WAGO Annual Meeting

June 16-18, 2011

Stein Eriksen Lodge

Park City, Utah

\title{
Zmiz1 is Overexpressed in Epithelial Ovarian Cancer and Associated with p53 Gene Mutations
}

Niyati J. Nadkarni, MD, ${ }^{1}$ Laura Rogers, BS, ${ }^{2}$ Megan Samuelson, MD, ${ }^{3}$ Traci Neff, BS, ${ }^{1}$ David P. Bender, MD, ${ }^{1,4}$ Amina Ahmed, MD, ${ }^{1,4}$ Koen DeGeest, MD, ${ }^{1,4}$ Anna Button, MS, ${ }^{5}$ Adam Dupuy, PhD, ${ }^{2}$ Michael J. Goodheart, MD ${ }^{1,4}$

Objectives: A recent transposon mutagenesis screen has identified the Zmiz1 gene as a new potential oncogene in carcinomas of the skin. It is well known that mutations of the p53 tumor suppressor gene are the most common genetic alterations in epithelial ovarian cancer. Previous studies have shown that Zmiz1 appears to transcriptionally regulate the p53 gene. There are no studies that evaluate Zmiz1 expression and alterations in the p53 gene in gynecologic malignancies, specifically epithelial ovarian cancer. We hypothesize Zmiz1 expression is elevated in epithelial ovarian tumors and there is an interaction between Zmiz1 and the p53 tumor suppressor gene in ovarian tumors.
Methods: A retrospective chart review was performed to identify patients diagnosed with epithelial ovarian cancer. Data collected included clinicopathologic factors, patient follow-up and mortality. Zmiz1 staining and p53 sequencing were performed on all patients. Statistical analyses included chi-square analysis and the binomial test for clinical and pathologic factors. The log rank test was used to evaluate survival analysis.

Results: Included in the analysis were sixty-one patients diagnosed with epithelial ovarian cancer. Immunostaining for the Zmiz1 protein was performed with the following results: $17(28 \%)$ tumors were positive, $11(18 \%)$ tumors had equivocal staining,

${ }^{1}$ Division of Gynecologic Oncology, Department of Obstetrics \& Gynecology, ${ }^{2}$ Department of Anatomy and Cell Biology, ${ }^{3}$ Department of Pathology, ${ }^{4}$ Holden Comprehensive Cancer Center, ${ }^{5}$ Department of Biostatistics, The University of lowa, lowa City, lowa 52242

Corresponding author: Michael Goodheart, Department of Obstetrics and Gynecology, University of lowa, 3234 MERF, 200 Hawkins Drive, lowa City, IA, 52242. Telephone(319) 335-2015 michael-goodheart@uiowa.edu medium, provided the original work is properly cited. 
WESTERN ASSOCIATION OF GYNECOLOGIC ONCOLOGISTS (WAGO)

WAGO Annual Meeting

June 16-18, 2011

Stein Eriksen Lodge

Park City, Utah

and $33(54 \%)$ tumors were negative. All of the benign ovarian specimens were negative for Zmiz1 immunostaining. Of the tumors with positive Zmiz1 staining, $13 / 17(76 \%)$ had p53 gene mutations $(p=0.03)$. The majority of patients without p53 mutations stained negative for Zmiz1 $(p=0.005)$. The overall survival for patients with Zmiz1 positive staining was $37 \%$ vs. $52 \%$ for patients with negative staining $(p=0.10)$.
Conclusions: Zmiz1 staining is elevated in patients with epithelial ovarian cancer. Tumors that are Zmiz1 positive are associated with mutations of the p53 gene. Zmiz1 overexpression may be associated with decreased survival in patients with epithelial ovarian cancer. Additional studies are needed to more clearly determine the role of Zmiz1 in patients with epithelial ovarian cancer.

Zmiz1, ovarian cancer and p53 mutations 